\section{Armin Studer}

\section{Nucleus prosthesis: a new concept}

concept of a compressible material encased by a strong, inelastic outer layer emerged as the logical conclusion. Specific requirements during operation and during the entire life of the implant have to be taken into consideration in developing new solutions. In this paper, the development of the nucleus prosthesis will be discussed and a new concept presented.

Keywords Low-back pain - Disc replacement - Nucleus prosthesis . Nucleus prolapse

\author{
A. Studer \\ Mathys Medical Ltd, \\ Güterstrasse 5, P.O. Box, \\ 2544 Bettlach, Switzerland \\ e-mail: armin.studer@mathysmedical.com, \\ Tel.: +41-32-6441751, \\ Fax: +41-32-6441173
}

\begin{abstract}
In a large number of lownucleus pulposus. Although the pathogenesis is still the topic of bate, the nucleus seems to play an gression of low-back in the proin its treatment. Numerous surgical treatment solutions have been patented or described in the literature; however, only a few seem promising and are in use today. After the development of numerous ideas, for instance metallic implants, the
\end{abstract}

\section{The nucleus: origin and target}

Origin of pain development

The nucleus pulposus is the central soft and gelatinous region of each intervertebral disc, surrounded by multilayered fibres of the annulus fibrosus. It is situated closer to the posterior border of the annulus fibrosus than to the anterior one [15]. The main organic structural components are collagens and proteoglycans. While collagens account for less than $20 \%$ of the dry weight of the central nucleus, proteoglycans represent as much as $50 \%$ in a child. The proteoglycans provide the tissue with its stiffness and resistance against compression by their interactions with water [6]. The presence of these hydrophilic proteins is the reason for the water-binding capacity of the nucleus (swelling pressure). The equilibrium of water content also depends on the external disc load. Increased loads squeeze water into the adjacent vertebral endplates. Unloading leads to return of water. This pumping mechanism provides the metabolism to the vessel-free nucleus [3]. Consequently, the fluid content of the nucleus shows diurnal 
variations. In the morning after rest, the turgor, and with it the thickness, is higher; by the evening the nucleus has lost some turgor.

The water content of the nucleus is highest in youth, at about $80 \%$, and decreases gradually with age [12]. It starts reducing in the fourth decade due to a gradual change in the type of proteoglycans [2]. Loss of height is the consequence. In an experimental study by Brinckmann and Grootenboer [5], for instance, about $1 \mathrm{~g}$ of tissue loss in the nucleus pulposus led to a loss of height of approximately $0.8 \mathrm{~mm}$. This influences the load sharing between nucleus pulposus, annulus fibrosus and facet joints. The decrease in interdiscal pressure reduces the engagement of the nucleus in the load flow, thus increasing the portions of the load carried by the annulus fibrosus and facet joints. Finally, it may result in damage to the joint cartilage, reactive hypertrophy and inflammation, and the neuroforaminal space of the exiting nerve roots may be impaired as well. The increasing load and the tissue alterations of the annulus fibrosus are the base for radial tears, cracks and fissures. The nucleus will finally prolapse if the mechanisms for self-healing are insufficient. The deformation of a nerve root clinically imposes radicular pain. In contrast, the irritation and inflammation of the facet joints lead to so-called pseudoradicular pain.

Since, in many cases, the nucleus pulposus seems to be the starting point of the degenerative cascade, it should also be the treatment target. But in the past, surgical interventions consisted either in a decompression of the nerve roots alone, in a fusion of motion segments or in a combination of both. All methods lead to an improvement in the clinical symptoms for a certain time. However, these methods do not solve the real problem. The problems are shifted to the adjacent motion segments because of the fusion or the contrasting instability of the segment [21].

\section{Treatment target}

The idea of restoring the biomechanical function of the disc by replacing only the nucleus as the cause of degeneration and pain seems very elegant, but has several limitations. The nucleus replacement is based on the assumption that the annulus and the endplates are still functioning properly [3]. This boundary condition has an important impact on patient selection and the indications for surgery. Potential patients are young and without many accompanying degenerative changes in the spine. Implanting an artificial substance into the spine of a young patient, however, raises questions about the longevity of the implant [13]. Wear problems, known from total hip replacements [20], and possible degradation processes within the nucleus material may lead to a need for re-intervention after 15-20 years. Furthermore, the risk of herniation of the new, artificial nucleus has to be minimised. The likelihood of this event should be lower than that of a normal prolapse [19]. The latter requirement has a direct impact on the surgical technique. While inserting the nucleus, the intraoperative damage to the annulus fibrosus must be kept to a minimum.

Numerous solutions have been patented or described in the literature, but only a few seem promising and are in use today. Initially attempts were made by injecting an acrylic mass [7, 9], and this was later followed by silicone [19]. Roy-Camille et al. used a latex bag to reduce the probability of material herniation and to decrease fatigue behaviour [18]. Nucleus replacement by means of metallic implants was attempted, but was abandoned because of migration and subsidence problems [8].

The concept of a compressible material, encased by a strong, inelastic outer layer, emerged as the logical consequence of the first ideas, and was first tested in vivo by Hou et al. [11], and brought to clinical use by Ray and Corbin [17]. However, clinical studies that prove the longterm efficiency of the device have yet to be done. More recent work is directed towards the development of materials (e.g. hydrogel) that better mimic the physiological behaviour of the nucleus $[13,16]$ and towards reducing invasiveness during insertion of the nucleus. This latter aim should be achieved by using devices with a memory effect. They are guided into the central disc cavity in a straight shape, but return to their pre-formed (in most cases spiral) structure $[4,14]$ once they are within the cavity. This solution offers two interesting features: the opening for the insertion of the implant is minimal, while the risk of a re-prolapse is reduced because of the overall size of the nucleus prosthesis after implantation. However, this exciting idea requires an excellent interaction between the materials used and the structure. The shear strength of the spiral's elastic contents, which is not an issue in homogeneous devices such as the PDN, becomes a major issue. Furthermore, delaminating between core and cover of the spiral has to be prevented. When using a hydrogel for the core, the cover sheath has to be guaranteed to remain intact.

\section{New treatment concept}

All above-mentioned requirements for a nucleus replacement were taken into consideration during the development of a new nucleus prosthesis design. Mathys Medical Ltd has realised a concept using a "snail" design for the final implant. A complex manufacturing process has been developed to specify the memory effect of the device, and to ensure that it has the required biomechanical properties such as shear strength, etc. The opening required by this specific design is minimal. Insertion into the disc is possible through a cross-stitched incision of the annulus. A tube is used to guide the straight-shaped prosthesis into the cleaned cavity of the disc. The insertion is possible through a percutaneous or minimal open posterior as well 
as a retroperitoneal approach. Once inside the empty cavity of the former nucleus, the device can return to its snail shape. Different lengths and diameters allow for compensation in disc height or diameter [1]. No intraoperative shortening of the device is necessary. The hydrogel of the nucleus prosthesis will start to swell after insertion. The prosthesis thus mimics diurnal changes and creep behaviour under mechanical load. As a consequence, the annulus fibrosus should be protected from overload. The core material possesses elastic and reversible plastic behaviour. Unlike other core materials such as polyurethane, nutrients can be transported via the nucleus to the annulus fibrosus. The materials used for both device components are biologically inert, to minimise adverse reactions.

The next steps towards a device for clinical use will include extensive biomechanical testing with respect to complex three-dimensional and fatigue behaviour. Next year, the biocompatibility will be tested before the first clinical studies. A clinical multicentre study will follow.

\section{Conclusions}

The newly developed nucleus prosthesis by Mathys Medical Ltd has biomechanical and biological properties that come very close to those of the original nucleus. The intraoperative damage to the surrounding tissue, especially the annulus fibrosus, is minimal, thus reducing the risk of herniation of the nucleus prosthesis. However, the indication for this device has to be established very carefully with respect to accompanying damage of the disc.

The proposed concept may find its place as a first even prophylactic - treatment option for disc herniation. Especially in young patients, it can offer a long-term solution without limiting further treatment options. If the degeneration of the affected disc continues, replacement of the whole disc or even a fusion is still possible at a later time.

\section{References}

1. Aharinejad S, Bertagnoli R, Wicke K, Firbas W, Schneider B (1990) Morphometric analysis of vertebrae and intervertebral discs as a basis of disc replacement. Am J Anat 189:69-76

2. Akeson WH, Woo SL, Taylor TK, Ghosh P, Bushell GR (1977) Biomechanics and biochemistry of the intervertebral discs: the need for correlation studies. Clin Orthop 129:133-140

3. Bao QB, McCullen GM, Higham PA, Dumbleton JH, Yuan HA (1996) The artificial disc: theory, design and materials. Biomaterials 17:1157-1167

4. Baumgartner W (1992) Intervertebral prosthesis. US patent $5,171,280$

5. Brinckmann P, Grootenboer H (1991) Change of disc height, radial disc bulge, and intradiscal pressure from discectomy. An in vitro investigation on human lumbar discs. Spine 16:641646

6. Buckwalter JA (1995) Aging and degeneration of the human intervertebral disc. Spine 20:1307-1314

7. Cleveland DA (1955) The use of methylacrylates for spinal stabilization after disc operations. Marquette Med Rev 20:62-64

8. Fernstrom U (1966) Arthroplasty with intercorporal endoprosthesis in herniated disc and in painful disc. Acta Chir Scand Suppl 357:154-159
9. Hamby WB, Glaser HT (1959) Replacement of spinal intervertebral discs with locally polymerizing methyl methacrylate. J Neurosurg 16:311-313

10. Hart LG, Deyo RA, Cherkin DC (1995) Physician office visits for low back pain. Frequency, clinical evaluation, and treatment patterns from a U.S. national survey. Spine 20:11-19

11. Hou TS, Tu KY, Xu YK, Li ZB, Cai AH, Wang HC (1991) Lumbar intervertebral disc prosthesis. An experimental study. Chin Med J (Engl) 104: 381-386

12. Humzah MD, Soames, RW (1988) Human intervertebral disc: structure and function. Anat Rec 220:337-356

13. Kadoya K, Kotani Y, Abumi K, Takada T, Shimamoto N, Shikinami Y, Kadosawa T, Kaneda K (2001) Biomechanical and morphologic evaluation of a three-dimensional fabric sheep artificial intervertebral disc: in vitro and in vivo analysis. Spine 26:1562-1569

14. Lin C-I (1998) Artificial intervertebral disk and method for implanting the same. US patent 5,716,416

15. Markolf KL, Morris JM (1974) The structural components of the intervertebral disc. A study of their contributions to the ability of the disc to withstand compressive forces. J Bone Joint Surg Am 56:675-687
16. Meakin JR, Reid JE, Hukins DW (2001) Replacing the nucleus pulposus of the intervertebral disc. Clin Biomech 16:560-565

17. Ray CD (1988) Percutaneous discectomy. A new day-surgical method for herniated lumbar discs. Minn Med 71: 485-488

18. Roy-Camille R, Saillant G, Lavaste F (1978) Experimental study of lumbar disc replacement. Rev Chir Orthop Reparatrice Appar Mot 64 [Suppl 2]: 106-107

19. Schneider PG, Oyen R (1974) Plastische Bandscheibenchirurgie. I. Mitteilung: Bandscheibenersatz im lumbalen Bereich mit Silikonkautschuk. Theoretische und experimentelle Untersuchungen. Z Orthop Ihre Grenzgeb 112:1078-1086

20. Urban RM, Jacobs JJ, Tomlinson MJ, Gavrilovic J, Black J, Peoch M (2000) Dissemination of wear particles to the liver, spleen, and abdominal lymph nodes of patients with hip or knee replacement. J Bone Joint Surg Am 82: 457-476

21. Weber H (1983) Lumbar disc herniation. A controlled, prospective study with ten years of observation. Spine 8:131-140 